\begin{tabular}{|c|l|}
\hline Title & Segmentation of multi-phase X-ray computed tomography images \\
\hline Author(s) & Kato, Masaji; Kaneko, Katsuhiko; Takahashi, Manabu; Kawasaki, Satoru \\
\hline Citation & $\begin{array}{l}\text { Environmental Geotechnics, 2(2), 104117 } \\
\text { https://doi.org/L0.1680/envgeo.13.00036 }\end{array}$ \\
\hline Issue Date & 2015-03 \\
\hline Doc URL & http://hdl.handle.net/2115/60727 \\
\hline Type & article \\
\hline File Information & envgeo2-0104_offprint.pdf \\
\hline
\end{tabular}

Instructions for use 


\section{Segmentation of multi-phase X-ray computed tomography images}

1 Masaji Kato DrEng

Assistant Professor, Faculty of Engineering, Hokkaido University, Sapporo, Japan

2 Manabu Takahashi DrEng

Chief Senior Researcher, Institute for Geology and Geoinformation, National Institute of Advanced Industrial Science and Technology (AIST), Tsukuba, Japan
$3 \quad$ Satoru Kawasaki DrEng

Associate Professor, Faculty of Engineering, Hokkaido University, Sapporo, Japan

$4 \quad$ Katsuhiko Kaneko DrEng

Professor, Faculty of Engineering, Hokkaido University, Sapporo, Japan
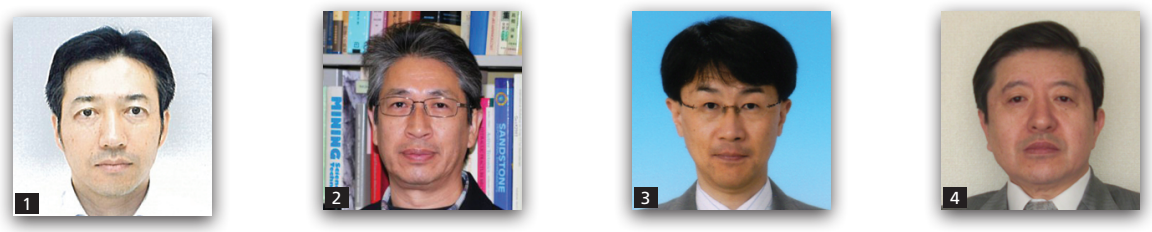

X-ray computed tomography (CT) is useful for non-destructively visualising internal features of non-transparent objects. In addition, it provides quantitative information about the geometry and spatial distribution of an object's constituent materials. However, X-ray CT images include blurs and noises. Herein, the authors focus on the partial volume effect that causes blurs depending on image resolution. Artificial materials (e.g., thin wire and threads) and natural materials (e.g., oil sand) were observed using a microfocus X-ray CT scanner. A maximum likelihood thresholding method considering the partial volume effect based on histogram data was applied for segmenting two- and three-phase X-ray CT images. Relative errors in measured cross-sectional areas of aluminium wires and nylon threads were evaluated for verifying the segmentation method. Occupancy ratios of each phase of oil sand to its bulk volume were quantified. The introduced segmentation method estimates the volume of each constituent of two- and three-phase porous materials with reasonable accuracy.

\section{Notation}

$a_{i}$

$B\left(m_{1}, m_{2}, \cdots, m_{n}\right)$

$f_{i}(x)$

$J_{0}(\mathrm{t}), J_{D}(\mathrm{t}), J_{Q}(\mathrm{t}), J_{K}(\mathrm{t})$

M

$M_{i_{1} i_{2} \cdots i_{n}}(x)$

$N\left(x ; \mu_{i}, \sigma_{i}^{2}\right)$

$N_{n_{p}}$

$n_{i}$

$\mathrm{t}$

$x$

$x_{p_{i}}$ area proportion or volume proportion of constituent class $i\left(0 \leq a_{i} \leq 1\right)$

multivariate beta function for $n$-class mixel

probability density function (PDF) of class $i$ within pure voxels

maximum likelihood thresholding criteria

total number of classes

PDF of $n$-class mixel for $n_{p}$-phase

materials $\left(2 \leq n \leq n_{p}\right)$

normal distribution function

total number of class classifications in

image of $n_{p}$-phase material

numbers of voxels within class $i$

threshold vector where the number of

vector elements equals $M-1$

intensity level

intensity level at peak of class $i$ in

histogram $x_{v}$

$\phi_{i}$

$\mu_{a}$

$\begin{array}{ll}\mu_{i} & \text { expectation of class } i \\ \tilde{\sigma}^{2} & \text { expectation of variance within each }\end{array}$

$\sigma_{a}^{2} \quad$ variance of mixel class

$\sigma_{i}^{2} \quad$ variance of class $i$

$\tilde{\sigma}_{k}^{2} \quad$ variance of class $k$

$\tilde{\omega}_{k} \quad$ occurrence probability of class $k$

\section{Introduction}

$\mathrm{X}$-ray computed tomography $(\mathrm{CT})$ is useful not only in medical fields but also in geotechnical engineering. The technique aids non-destructive, non-disturbed observations of the internal structures of geomaterials such as soil and rocks. Through CT observations and subsequent calculations, greyscale CT images are obtained. Important information, hidden at times, obtained from 


\section{Offprint provided courtesy of www.icevirtuallibrary.com Author copy for personal use, not for distribution}

the images should be extracted. Segmentation of CT images is one of the simplest methods for investigating material properties. The segmentation of dried soil and rocks (similarly, water-saturated soil and rocks) consisting of solid (minerals) and fluid (air or water in pores) phases is a challenging problem, and it is only recently that robust methods have been proposed for dealing with the challenges imposed by soil (Houston et al., 2013; Schlüter et al., 2010) and rock images (Kato et al., 2013). The image segmentation of such two-phase materials is related to problems associated with storage of the materials and fluid flow through them. Practically, three-phase materials consisting of the solid, liquid and gaseous phases exist and need to be segmented. In this study, the focus is on materials consisting of two or more phases, also called multiphase materials.

X-ray CT images of multi-phase materials are different from their real images because of the sampling and quantisation involved in generating digital images (Figure 1). Some level of blur is inevitable in both X-ray CT images and optical images. There are two primary causes of blur. The first is the penumbra effect, which depends on the focal spot size and the distances between the X-ray source, the object and the detector (Curry et al., 1990). The second is the partial volume effect, which is ascribed to the existence of multiple substances in each voxel of a CT image (Ketcham and Carlson, 2001). The blur resulting from these effects can be minimised if the focal spot size can be reduced. This leads to enhanced spatial resolution, which is equivalent to minimising the voxel size. However, despite this, blur is not eliminated.

The partial volume effect appears intrinsically, without exception, in digital images captured using any optical instrument. This effect appears not only on the slice plane, shown in Figure 1, but also along the thickness direction (normal to the plane). The partial volume effect is a persistent problem in three-dimensional (3D) digital images of multi-phase materials.

To tackle this issue, Choi et al. (1991) introduced and applied the concept of mixed pixel, or mixel, to the classification of medical magnetic resonance images of the brain. Numerous studies have focused on the partial volume effect or mixels in various fields such as medical science (Kim et al., 2005; Shattuck et al., 2001; Tohka et al., 2004), remote sensing (Kageyama and Nishida, 2004; Okamoto and Fukuhara, 1996), soil and rock engineering (Kato et al., 2013; Oh and Lindquist, 1999) and information technology (Kitamoto and Takagi, 1998a, 1998b, 1999, 2000). Mixels contain multiple-phase constituents within a single pixel (or voxel), leading to image blurring (Figure 2). Conversely, pixels (or voxels) with only a single phase are called pure pixels (or pure voxels). Even in high-resolution digital images, there exist some mixels (Figure 3). The higher the image resolution, the lower the mixel/pixel (or mixel/voxel) ratio, which is the ratio of the number of mixels to the total number of voxels in an image and is shown numerically in the caption in Figure 3. However, mixels are not eliminated practically.

Numerous thresholding techniques are described in the literature (see reviews in Pal and Pal, 1993; Sahoo et al., 1988; Sezgin and Sankur, 2004). Iassonov et al. (2009) presented an overview of the thresholding techniques applied in recent porous media research. However, the performance of each technique depends on the purpose and the object of analysis. Baveye et al. (2010) reported difficulty in applying thresholding to soil images and X-ray CT data and that the outcome depended on the observer. Therefore, there exist several binarised image patterns of the object observed by different researchers. The thresholding of digital images of two-phase objects considering the partial volume effect was originally introduced for satellite images by Kitamoto (1999) and was demonstrated for X-ray CT images of packed glass beads and sandstone by Kato et al. (2013). Thresholding digital images of materials with more than two phases was addressed by several groups (Bhattad et al., 2010; Kumar et al., 2010; Vinegar and Wellington, 1987). However, the partial volume effect has not been considered in any such study conducted thus far.

Oil sand is a non-conventional energy resource, and major oil sand deposits have been discovered in Alberta, Canada, and Orinoco,

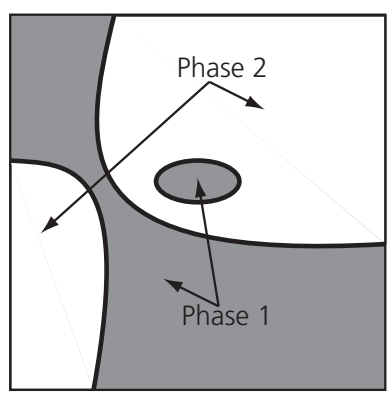

(a)

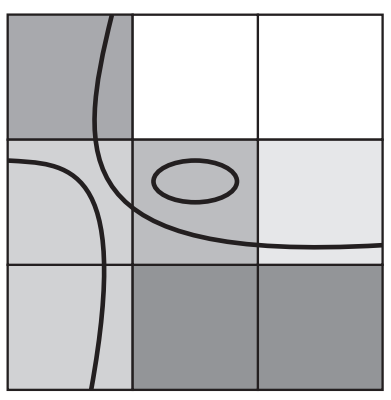

(b)

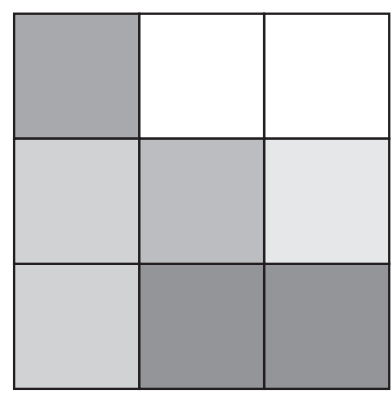

(c)
Figure 1. Schematic of image acquisition. Greyscale digital images are made from a two-phase substance owing to sampling and quantisation. (a) is a real image and (b) and (c) are its quantised versions taking into account the partial volume effect, where boundary lines are remaining in (b) 


\section{Offprint provided courtesy of www.icevirtuallibrary.com Author copy for personal use, not for distribution}

\begin{tabular}{|c|c|c|}
\hline $\begin{array}{c}\text { Class 3 } \\
\text { (Mixel) }\end{array}$ & Class 2 & Class 2 \\
\hline $\begin{array}{c}\text { Class 3 } \\
\text { (Mixel) }\end{array}$ & $\begin{array}{c}\text { Class 3 } \\
\text { (Mixel) }\end{array}$ & $\begin{array}{c}\text { Class 3 } \\
\text { (Mixel) }\end{array}$ \\
\hline $\begin{array}{c}\text { Class 3 } \\
\text { (Mixel) }\end{array}$ & Class 1 & Class 1 \\
\hline
\end{tabular}

(a)

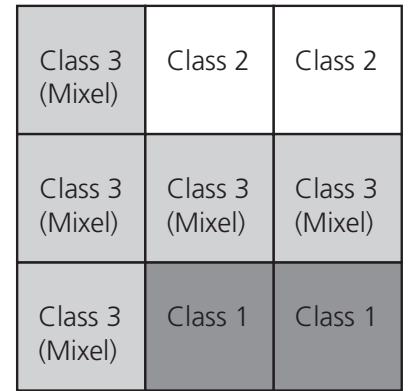

(b)
Figure 2. Digital images corresponding to Figure 1. (a) Original greyscale image and (b) image segmented with classes 1 and 2 representing pure pixels and class 3 representing their mixel

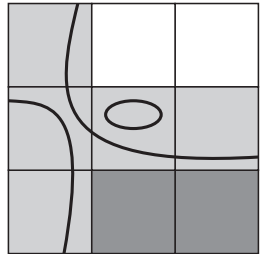

(a)

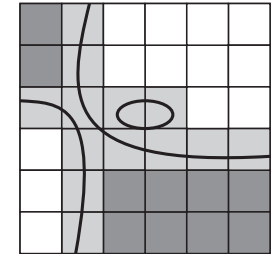

(b)

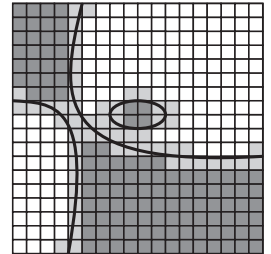

(c)
Figure 3. Partial volume effect according to the resolution of digital images made from image shown in Figure 1(a). (a) Low resolution, (b) intermediate resolution and (c) high resolution. The mixel/voxel ratios for the above resolutions are (a) 0.56 , (b) 0.36 and (c) $0 \cdot 17$, respectively

Venezuela (Sekiguchi, 2006). Oil sand is a mixture of sand grains (siliceous material), water, bitumen (extra-heavy oil) and methane gas. The volume fraction of natural bitumen in oil sand is one of the parameters for evaluating extra-heavy oil reserves. X-ray CT can be used for determining oil's internal structure as well as for basic data acquisition in the process of evaluating oil reserves. Oil sand samples are considered three-phase materials consisting of solid (sand grains), liquid (water and bitumen) and gaseous (air and methane) phases. Although bitumen does not act as a fluid at room temperatures, it corresponds to the liquid phase because its density is only slightly less than that of water. This ensures that there is a slight difference between the X-ray attenuation coefficients of water and bitumen.

In this study, the partial volume effect in X-ray CT images of multi-phase materials was modelled stochastically using Gaussian distribution for the different phases and beta distribution for the partial volume effect. In addition, an automatic image segmentation technique that considers the partial volume effect for CT imaging of porous materials was introduced. Then, the applicability of the developed segmentation technique was verified using two- and three-phase materials, including artificial materials such as metal wires and nylon threads as well as natural materials such as oil sand.

\section{Segmentation method}

Mixture model considering partial volume effect

For a multi-phase object, the spatial distribution data of its X-ray attenuation coefficients are obtained by X-ray CT scanning. Using these data, a greyscale image is drawn as a mixture of pure voxels and mixels (Figure 1). Histograms of the intensity level of CT images generated by scanning two-phase materials show either bimodal or unimodal distribution, and those generated using three-phase materials show either trimodal, bimodal or, occasionally, unimodal distribution. It is apparent that histograms are not generated only by the simple superposition of normal distributions, such as the clearly bimodal distribution of two-phase images and the clearly trimodal distribution of three-phase images, because of the partial volume effect.

In fact, the histogram of a class of pure voxels obeys a normal distribution because of slight inhomogeneities within a given one-phase substance and minute variations in the dispersion and absorption of X-rays. Accordingly, the probability density function (PDF) $f_{i}(x)$ of a constituent class $i$, corresponding to the pure voxels of phase $i$, can be expressed as follows

1.

$$
f_{i}(x)=N\left(x ; \mu_{i}, \sigma_{i}^{2}\right)=\frac{1}{\sqrt{2 \pi \sigma_{i}^{2}}} \exp \left\{-\frac{\left(x-\mu_{i}\right)^{2}}{2 \sigma_{i}^{2}}\right\}(i=1,2, \cdots)
$$

where $N\left(x ; \mu_{i}, \sigma_{i}^{2}\right)$ denotes the normal distribution function for intensity level $x$, expectation $\mu_{i}$ and variance $\sigma_{i}^{2}$ of constituent class $i$. For two-phase materials, $i$ takes the integer value of either 1 or 2 . For three-phase materials, $i$ takes an integer value between 1 and 3 . In both cases, the maximum value of $i$ equals the number of phases.

Mixels, which correspond to voxels occupied by two or more phases, appear inevitably in a digital image. Two-class mixels (voxels occupied by two phases) and three-class mixels (voxels occupied by three phases) are required for the segmentation of two- and three-phase materials in this study. Two- and three-class mixels are explained below, and multi-class mixels are generalised in Appendix 1.

For two-phase materials, one type of two-class mixels exists that corresponds to pixels (or voxels) occupied by phases 1 and 2 . The area proportion distribution of this class, which is defined by Kitamoto and Takagi $(1998 \mathrm{~b}, 2000)$ as the proportion of the area that each constituent class occupies within a mixel in $2 \mathrm{D}$ images, is assumed to obey beta distribution. It has also been stated that the beta distribution has adequate potential for describing various types of area proportion distributions such as uniform-shaped, U-shaped, J-shaped, L-shaped and bell-shaped. In this study, the area proportion in two dimensions is expanded to volume proportion in three dimensions. The PDF of two-class mixels of two-phase materials is expressed as follows

2. $\quad M_{12}(x)=\frac{1}{B\left(m_{1}, m_{2}\right)} \int_{0}^{1} a_{1}^{m_{1}-1} a_{2}^{m_{2}-1} N\left(x ; \mu_{a}, \sigma_{a}^{2}\right) \mathrm{d} a_{1}$ 
where $B\left(m_{1}, m_{2}\right)$ denotes the bivariate beta function, which is given by the following equation

3.

$$
B\left(m_{1}, m_{2}\right)=\int_{0}^{1} a_{1}^{m_{1}-1} a_{2}^{m_{2}-1} \mathrm{~d} a_{1}
$$

where $a_{i}$ is the volume proportion of constituent class $i\left(0 \leq a_{i} \leq 1\right)$, and the relation $a_{1}+a_{2}=1$ holds; parameters $m_{1}$ and $m_{2}$ are greater than 0 ; and $\mu_{a}$ and $\sigma_{a}^{2}$ are given as follows

4. $\mu_{a}=a_{1} \mu_{1}+a_{2} \mu_{2}$

5. $\sigma_{a}^{2}=a_{1}^{2} \sigma_{1}^{2}+a_{2}^{2} \sigma_{2}^{2}$

Accordingly, for two-phase materials, each pure voxel can be assigned to classes 1 and 2 , and the two-class mixel to class 3 .

For an image of a three-phase material, more than one type of mixel is needed. It is possible to choose a pair from among three phases or to consider all three. Accordingly, the mixels in an image of a three-phase material can be classified into four types: three types of pairs from among three phases and one type for all three phases. The PDFs of two-class mixels of three-phase materials are obtained by replacing $a_{1}, a_{2}$ and $M_{12}(x)$ with $a_{i 1}, a_{i_{2}}$ and $M_{i i_{2}}(x)$, respectively, where $i_{1}, i_{2} \in 1,2,3$ and $i_{1}<i_{2}$.

The PDFs of three-class mixels of three-phase materials are expressed as follows

6.

$$
M_{123}(x)=\frac{1}{B\left(m_{1}, m_{2}, m_{3}\right)} \int_{0}^{1} \int_{0}^{1} a_{1}^{m_{1}-1} a_{2}^{m_{2}-1} a_{3}^{m_{3}-1} N\left(x ; \mu_{a}, \sigma_{a}^{2}\right) \mathrm{d} a_{1} \mathrm{~d} a_{2}
$$

where the trivariate beta function is

$$
\text { 7. } \quad B\left(m_{1}, m_{2}, m_{3}\right)=\int_{0}^{1} \int_{0}^{1} a_{1}^{m_{1}-1} a_{2}^{m_{2}-1} a_{3}^{m_{3}-1} \mathrm{~d} a_{1} \mathrm{~d} a_{2}
$$

where $a_{i}$ is the volume proportion of constituent class $i\left(0 \leq a_{i} \leq 1\right)$, such that the relation $\sum_{i=1}^{3} a_{i}=1$ holds; parameters $m_{1}, m_{2}$ and $m_{3}$ are greater than 0 ; and $\mu_{a}$ and $\sigma_{a}^{2}$ are as follows

8. $\mu_{a}=\sum_{i=1}^{3} a_{i} \mu_{i}$

9.

$$
\sigma_{a}^{2}=\sum_{i=1}^{3} a_{i}^{2} \sigma_{i}^{2}
$$

Here, by assuming a few aspects of the phase distribution, the authors can simplify the problem and apply the model for solving it.

\section{Thresholding method}

First, the scope of investigation was limited to two-class segmentation problems with a bimodal histogram obtained from digital images. Kitamoto $(1999,2000)$ applied a mixel model for distinguishing between the cloud and sea phases in satellite images. In this study, the maximum likelihood thresholding method proposed by Kitamoto (1999), which considers the effect of mixels, was adopted for two-class segmentation problems.

For two-class segmentation problems with a bimodal histogram, the total number of classes $M$ is three (two for pure voxels and one for mixels), and two thresholds are employed: one between classes 1 and 3 , and another between classes 2 and 3 . These thresholds are referred to as $t_{1}$ and $t_{2}$, respectively, and can be expressed by the vector $\mathbf{t}=$ $\left(t_{1}, t_{2}\right)$, where the number of vector components equals $M-1$. The maximum likelihood thresholding criteria used in this study are functions of the threshold vector $\mathbf{t}$ along with the associated statistical properties of each class and are shown in Appendix 2.

After obtaining histogram data from the images, the threshold vector $\mathbf{t}$ was selected according to the following steps.

- Check whether the histogram exhibits bimodal distribution geometrically.

- Set the intensity levels to $x_{p 1}$ and $x_{p 2}$ at the left and right peaks (where $x_{p 1}<x_{p 2}$ ), respectively, and to $x_{v}$ in the trough between the two histogram peaks temporarily. The threshold vector components $t_{1}$ and $t_{2}$ should be between $x_{p 1}$ and $x_{v}$ and between $x_{v}$ and $x_{p 2}$, respectively.

- Calculate the logarithmic likelihood shown in Appendix 2 , which is a function of threshold vector $\mathbf{t}$ along with the associated statistical properties of each class. Given the threshold vector $\mathbf{t}$, the expectations, variances and occurrence probabilities of classes 1 and 2 for pure voxels are calculated from the histogram data. Then, based on these statistical values, the stochastic parameters of class 3 for mixels are obtained analytically using Equations 2-5. Here, for simplicity, the parameters $m_{1}$ and $m_{2}$ in the beta function are set to 1 under the assumption that the volume proportion has a uniform-shaped distribution because the boundary between two phases of a material is simple and smooth.

- Accordingly, determine the optimum threshold vector $\mathbf{t}$ in the possible range to maximise the above-mentioned likelihood.

The authors automated the above procedure for the selection of threshold values. The occurrence probabilities and variances of classes 1 and 2 in the digital images were unknown before thresholding. Accordingly, a likelihood criterion $J_{K}(\mathbf{t})$ was selected and tested as the first thresholding criterion (see Appendix 2). However, thresholding in this manner sometimes resulted in both thresholds being almost the same. In such cases, the authors applied other criterion, such as $J_{D}(\mathbf{t})$. 


\section{Offprint provided courtesy of www.icevirtuallibrary.com Author copy for personal use, not for distribution}

The above-mentioned thresholding method can be applied to multiphase digital images. However, the challenge in doing so is that the number of thresholds would increase with an increase in the number of phases (see Appendix 1). In this case, by adopting some suppositions of spatial phase distribution for an image, the problem can be simplified. For example, oil sand is composed of the solid (sand grain), liquid (water and bitumen) and gaseous (methane gas and air penetrating after coring) phases. The bitumen remains on the surfaces of the sand grains and in the spaces between the sand grains. Therefore, the liquid phase wets the surface of the solid phase, and the gaseous phase occupies the remainder of the pores' volume, as shown in Figure 4. A few mixels consist of the solid and gaseous phases or all three phases.

Figure 5 shows an example of the histogram generated from the three-phase image for oil sand. In this figure, the lines show the probability distributions of five classes (three for pure voxels and two for mixels), and their superposition, which corresponds to the histogram. The mixel types that consist of the solid and gaseous phases as well as all three phases are lacking, as mentioned above.

\section{Calculation of volume or occupancy ratio}

Once the thresholds are set, the volume (or cross-sectional area) and occupancy ratio, which is the ratio of object phase volume to total volume of porous material, can be evaluated. For two-phase images, the ratio of all three classes (two classes for pure voxels and one class for mixels) was obtained. The area (volume) of mixels must eventually be divided into two phases for evaluating the occupancy ratio. It is reasonable to assume that the area of mixels can be divided into two phases according to the ratio of the numbers of voxels in classes 1 and 2 (Kato et al., 2008, 2013; Kobayashi et al., 2009, 2010). Thus, the occupancy ratio $\phi_{1}$ of phase 1 can be calculated as follows

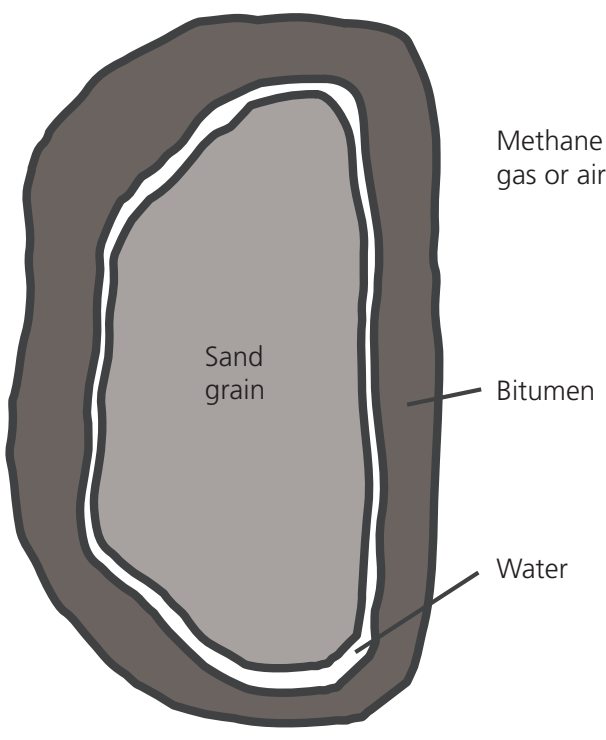

Figure 4. Schematic of spatial distribution and contact between each constituent in oil sand

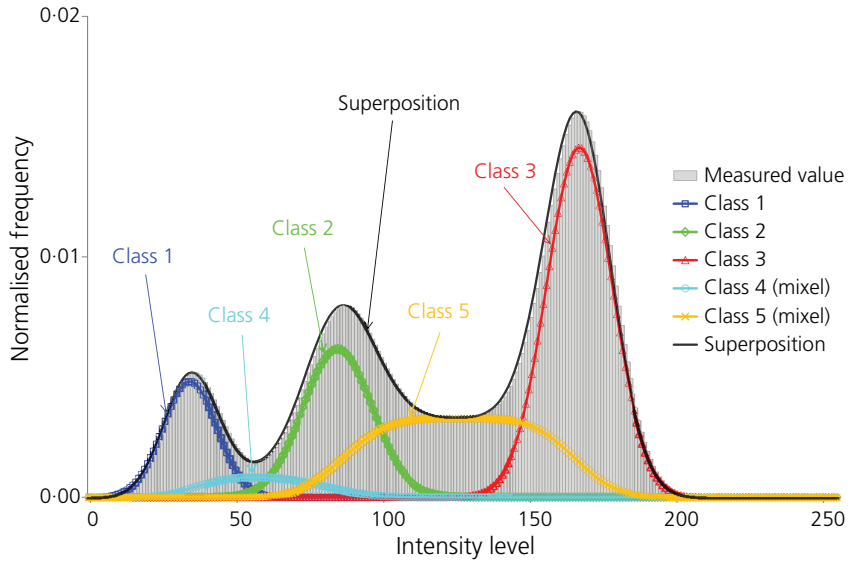

Figure 5. Example of histogram obtained from a three-phase image with lines showing the probability distributions of five classes and their superposition

10. $\phi_{1}=\frac{n_{1}}{n_{1}+n_{2}}$

where $n_{1}$ and $n_{2}$ are the numbers of voxels in classes 1 and 2, respectively. Note that the number of mixels (i.e., voxels in class 3) does not appear in this calculation (Kato et al., 2013). The occupancy ratio of phase 2 can be evaluated in the same manner.

The above-mentioned calculation can also be applied to the images of multi-phase materials. For example, in three-phase images, the occupancy ratio of phase 1 in porous material $\phi_{1}$ can be calculated on the basis of a few assumptions regarding spatial phase distribution in the image using the following equation

11. $\phi_{1}=\frac{n_{1}}{n_{1}+n_{2}+n_{3}}$

where $n_{1}, n_{2}$ and $n_{3}$ are the numbers of voxels in classes 1,2 and 3, respectively. Note that the numbers of mixels (i.e., voxels in classes 4 and 5) do not appear in this calculation. The occupancy ratio of another phase can be evaluated in the same manner.

\section{X-ray CT}

Microfocus X-ray CT

$\mathrm{X}$-ray $\mathrm{CT}$ is a non-destructive, non-invasive 3D visualisation and quantification tool for non-transparent objects. Microfocus $\mathrm{X}$-ray CT is based on recording X-ray projections of an object at different angles and stacking several sequential slices. A filtered back-projection algorithm is then used for reconstructing a slice image through the object to reveal the linear attenuation coefficient distribution. The attenuation coefficient depends on the applied 


\section{Offprint provided courtesy of www.icevirtuallibrary.com Author copy for personal use, not for distribution}

$\mathrm{X}$-ray energy, and the atomic number and density of a given object. Descriptions of microfocus X-ray CT instruments and reconstruction algorithms can be found in the work of Kak and Slaney (1988).

In this study, a microfocus X-ray CT scanner (TOSCANER 31300 $\mu$ hd, Toshiba IT and Control Systems Co.) installed at Hokkaido University, Japan, was used (Kato et al., 2009, 2013; Kawaragi et al., 2009; Kobayashi et al., 2009, 2010; Yamanaka et al., 2011). The focal spot size of the X-ray source assembly is $5 \mu \mathrm{m}$. Scans were conducted at $130 \mathrm{kV}$ (the maximum tube voltage of the device) and $62 \mu \mathrm{A}$. Furthermore, the full-scan mode of a single slice was selected for this study. It is possible to set the number of views (number of angular projections, $\leq 4800$ ) and number of stacks per angle (number of exposures per frame, $\leq 50$ ) arbitrarily. For this study, 1500 views and 20 stacks per angle were selected. In addition, the distance between the focal spot of the X-ray source and the centre of rotation (focus-centre distance (FCD)) can be adjusted $(\leq 50 \mathrm{~cm})$ for varying $\mathrm{CT}$ image resolution.

In this study, a tiny elongated voxel with cross-sectional dimensions of $6.3 \mu \mathrm{m} \times 6.3 \mu \mathrm{m}$ for slice scan and $10.7 \mu \mathrm{m} \times 10.7 \mu \mathrm{m}$ for cone beam scan, respectively, and a voxel height of approximately $20 \mu \mathrm{m}$

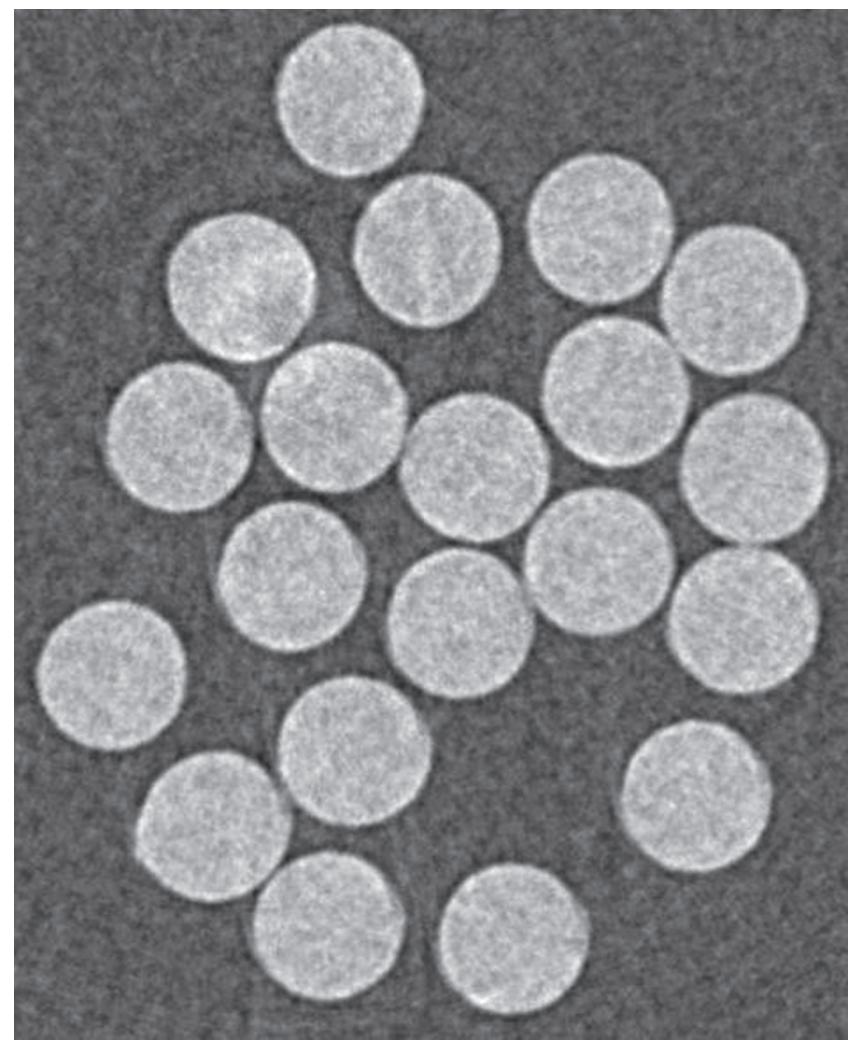

(a)

Figure 6. (a) Original X-ray CT image of only nylon threads (size $3.7 \times 3.0 \mathrm{~mm}$ ) and (b) image segmented using thresholding method based on mixel model; black regions represent class 1 depending on the FCD, were used. The matrix size was set to 2048 $\times 2048$ and $1024 \times 1024$ pixels for slice scan and cone beam scan, respectively.

The gain and position of the CT scanner's rotational centre were calibrated carefully for reducing artefacts and obtaining clear images. However, residual blur is inevitable in X-ray CT images. There are two primary causes of blur: the penumbra effect, which depends on focal spot size and distances between the X-ray source, object and detector (Curry et al., 1990); and the partial volume effect, which results from the existence of multiple substances in each voxel of the CT images (Ketcham and Carlson, 2001). In this study, the partial volume effect was modelled stochastically, and the developed model was used for image segmentation.

\section{Samples}

The authors used artificial and natural porous materials. The artificial materials were thin line shapes such as aluminium wires and nylon threads. The diameter and density of these objects were $0.50 \mathrm{~mm}$ and $2.65 \mathrm{~g} / \mathrm{cm}^{3}$ (aluminium wire), and $0.52 \mathrm{~mm}$ and $1.5 \mathrm{~g} / \mathrm{cm}^{3}$ (nylon thread). These objects were homogeneous, and their densities were close to those of geomaterials. Furthermore, the cross-sectional areas of these objects were constant throughout

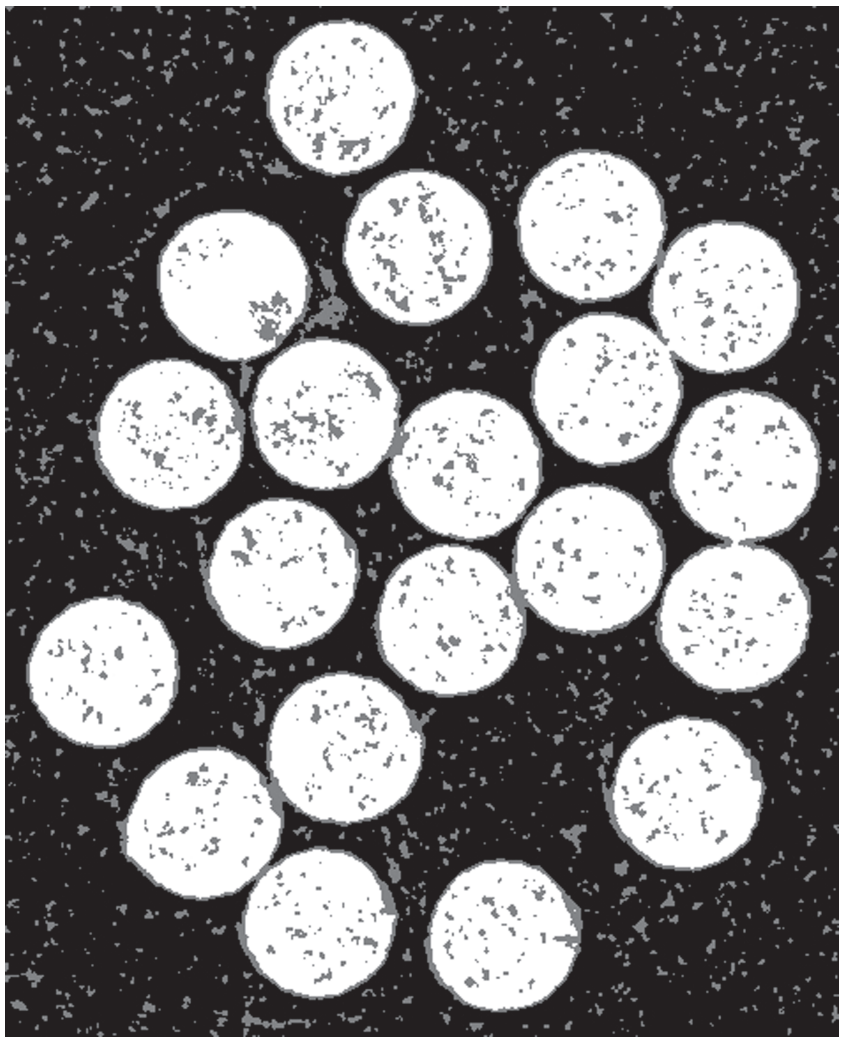

(b)

(air), white regions represent class 2 (nylon threads) and grey regions represent class 3 (mixels) 
their lengths. For observations, bundles comprising 20 wires of only one type as well as bundles of ten wires each of both aluminium and nylon were used.

The natural material used was an oil sand sample cored from a depth of $284 \mathrm{~m}$ in a part of the Hangingstone area in Athabasca, Alberta, Canada. This sample was preserved by freezing after coring. However, it was assumed that the volatile constituent of crude oil in the sample had evaporated. Bitumen remained on the surface of the sand grains and in the spaces between them. The oil sand sample comprised sand grains, bitumen, water and air. The liquid phase (bitumen and water) wetted the surface of the solid phase (sand grains). The gaseous phase existed in the remainder of the inter-grain volume. The oil sand sample was cut into approximately $1-\mathrm{cm}^{3}$ pieces for CT scanning.

In this study, sand grain was considered as the solid phase, water and bitumen as the liquid phase and air trapped in the pores as the gaseous phase. However, bitumen was not in the liquid state at room temperature.

\section{Results and validation}

\section{Artificial materials}

Bundled thin lines (aluminium wires and nylon threads separately) were scanned using an X-ray CT scanner. For example, the original CT image of bundled nylon threads is shown in Figure 6(a). Its size is mentioned in the corresponding figure caption. In this figure, light grey circles represent nylon threads. The number of threads is 20. The histogram of this image is shown in Figure 7. The histogram has a bimodal distribution, thus implying that the image is of a two-phase material. Using this histogram along with the procedure for setting the threshold vector $\mathbf{t}$, explained above ('Thresholding method'), two types of logarithmic likelihood distributions corresponding to threshold vector $\mathbf{t}$ were obtained, as

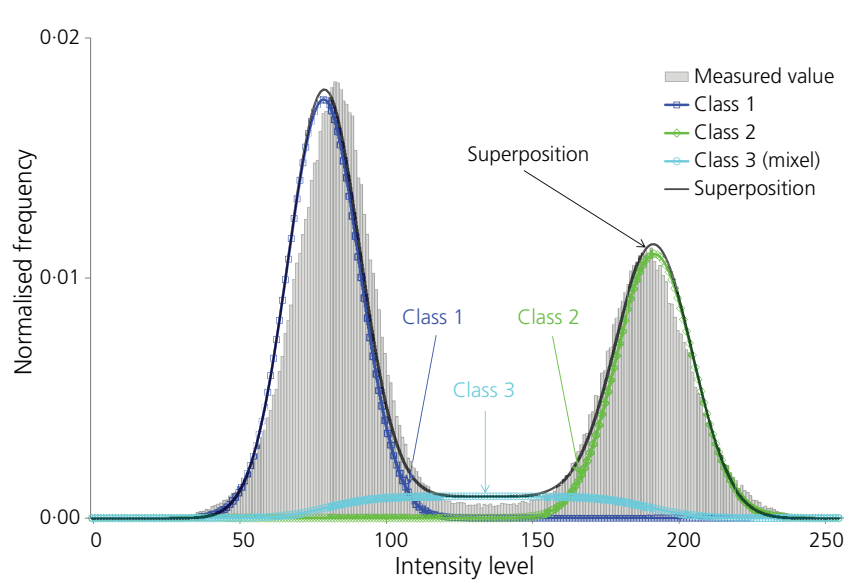

Figure 7. Histogram of CT image of nylon threads in Figure 6(a). The lines indicate the probability distributions of three classes and their superposition shown in Figure 8. The authors found that the $J_{K}(\mathbf{t})$ criterion was unsuitable because both thresholds were almost the same at the point of maximum $J_{K}(\mathbf{t})$. Therefore, the $J_{D}(\mathbf{t})$ criterion was adopted instead of the $J_{K}(\mathbf{t})$ criterion, and the thresholds were determined at the point of maximum $J_{D}(\mathbf{t})$. In Figure 7 , the lines indicate the probability distribution of three classes (two of pure voxels and one of a mixel) calculated using the thresholds and their superposition. After applying the thresholding method based on the mixel model, described above ('Segmentation method'), to the histogram, the segmented image shown in Figure 6(b) was obtained. Using the

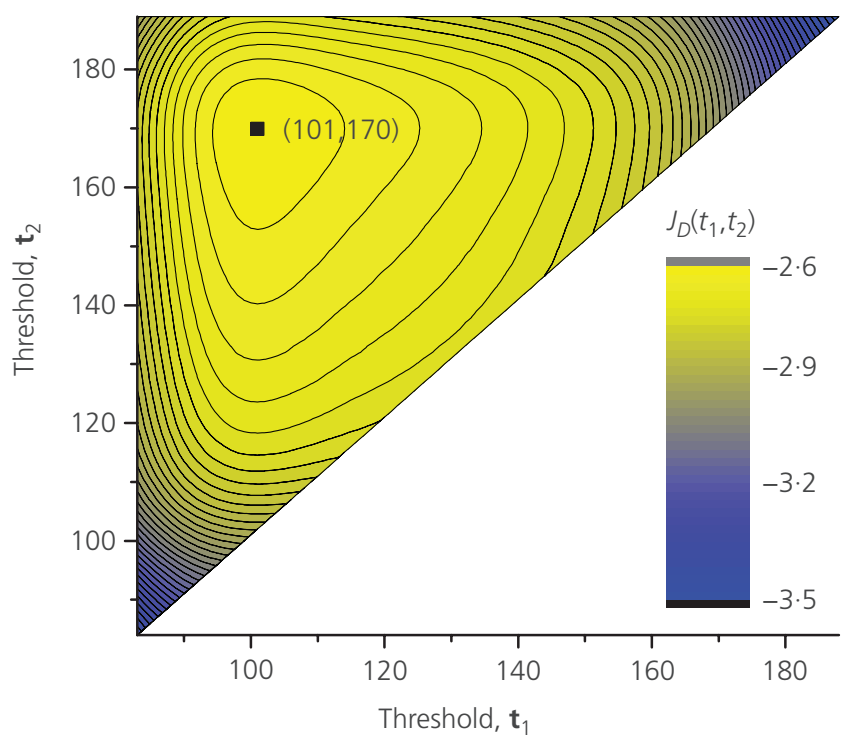

(a)

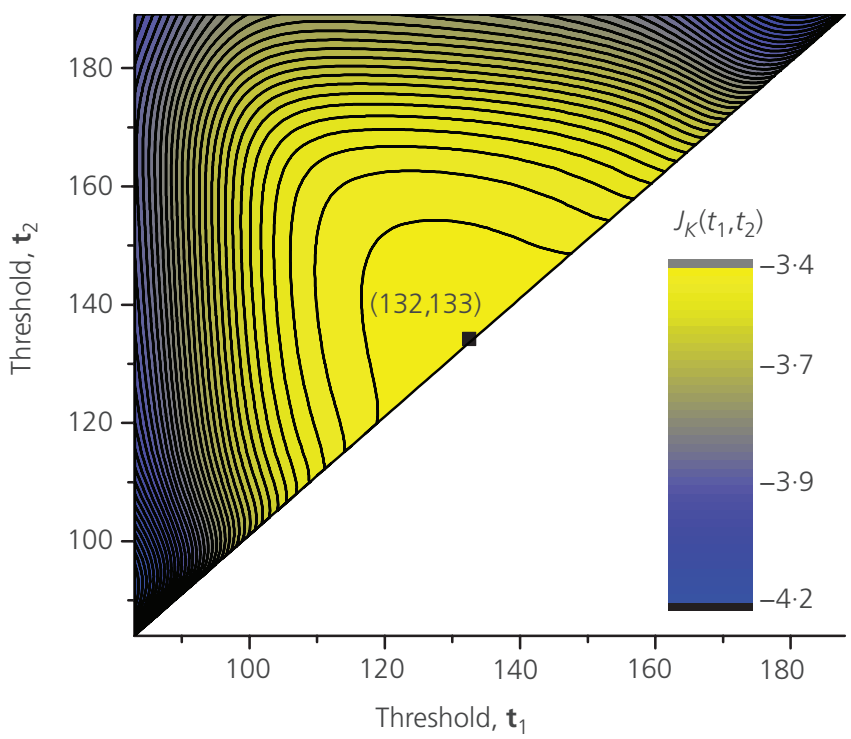

(b)

Figure 8. Logarithmic likelihood distributions corresponding to threshold vector $\mathbf{t}$ : (a) $J_{D}(\mathbf{t})$ and $(\mathrm{b}) J_{K}(\mathbf{t})$ for regions where $x_{p 1}<x_{p 2}$ with $t_{1}<t_{2}$. All given threshold value couples are for the point of maximum likelihood 


\section{Offprint provided courtesy of www.icevirtuallibrary.com Author copy for personal use, not for distribution}

\begin{tabular}{lccc}
\hline Material & $\begin{array}{c}\text { Actual cross-sectional } \\
\text { area: } \mathrm{mm}^{2}\end{array}$ & $\begin{array}{c}\text { Area estimated by this } \\
\text { segmentation method: } \mathrm{mm}^{2}\end{array}$ & Relative error \\
\hline Nylon threads & 4.31 & 4.42 & 0.03 \\
Aluminium wires & 4.12 & 4.50 & 0.09 \\
Table 1. Comparison of cross-sectional areas in two-phase & & \\
segmentation & & \\
\end{tabular}

segmented image, the authors calculated the cross-sectional areas of the nylon threads and aluminium wires (Table 1).

Next, a bundle comprising ten aluminium wires and ten nylon threads was scanned. The original X-ray CT image is shown in Figure 9. Its size is mentioned in the corresponding figure caption. In the figure, the black regions represent air, the white regions represent aluminium wires and the grey regions represent nylon threads. The histogram of this CT image is shown in Figure 10. The histogram has a trimodal distribution, thus implying that this image is of a three-phase material. The lines in Figure 10 indicate the probability distributions of five classes (three of pure voxels and two of mixels) and their superposition. Figure 11 shows the image segmented using the thresholding method based on the mixel

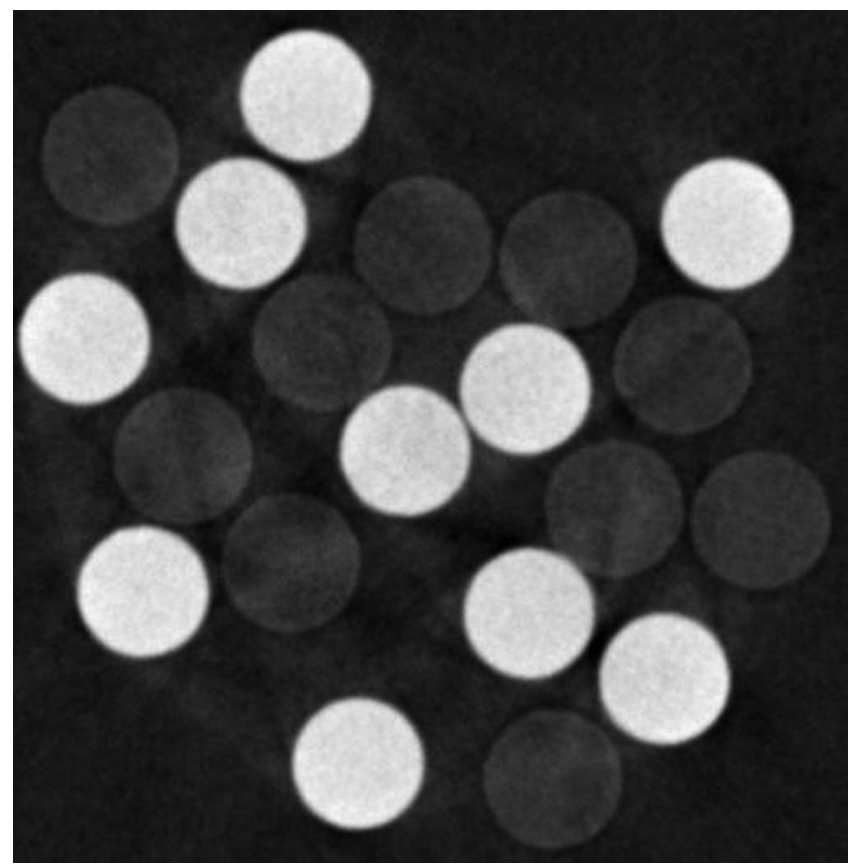

Figure 9. Original $X$-ray CT image of nylon threads and aluminium wires (size $3.2 \times 3.3 \mathrm{~mm}$ ); black regions represent air, white regions represent aluminium wires and grey regions represent nylon threads model for oil sand. Using the segmented image, the cross-sectional area of each constituent in a bundle comprising nylon threads and aluminium wires was calculated (Table 2).

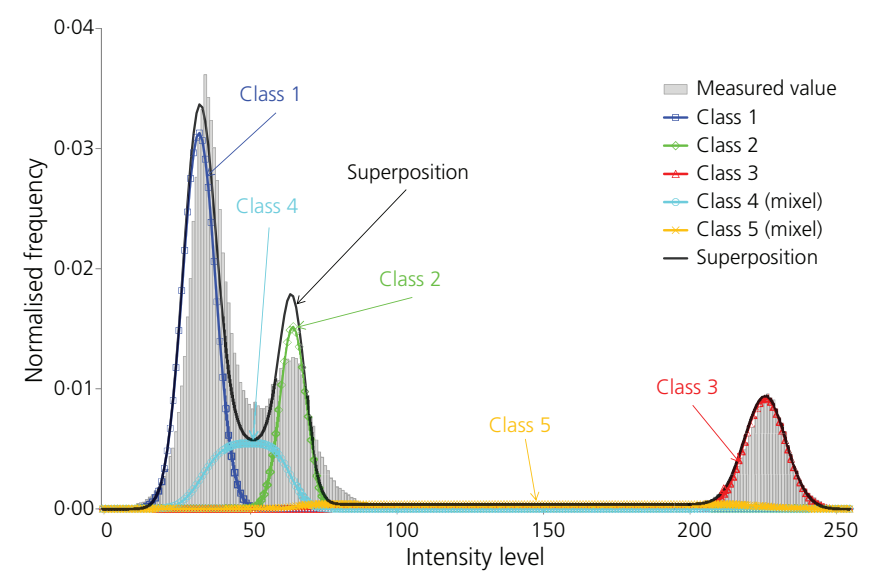

Figure 10. Histogram of the CT image of nylon threads and aluminium wires shown in Figure 9. The lines indicate the probability distributions of five classes and their superposition

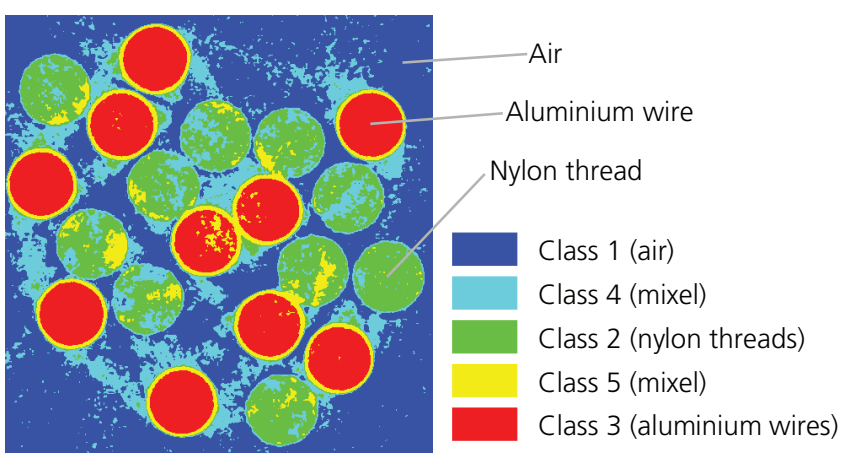

Figure 11. Image segmented using thresholding method based on the mixel model; blue regions represent class 1 (air), green regions represent class 2 (nylon threads), red regions represent class 3 (aluminium wires), sky blue regions represent class 4 (mixel of classes 1 and 2) and yellow regions represent class 5 (mixel of classes 2 and 3) 


\begin{tabular}{lccc}
\hline Material & $\begin{array}{c}\text { Actual cross-sectional } \\
\text { area: } \mathrm{mm}^{2}\end{array}$ & $\begin{array}{c}\text { Area estimated by this } \\
\text { segmentation method: } \mathrm{mm}^{2}\end{array}$ & Relative error \\
\hline Nylon threads & 2.12 & 2.57 & 0.21 \\
Aluminium wires & 1.96 & 2.04 & 0.04 \\
Air & 6.41 & 5.89 & 0.08
\end{tabular}

Table 2. Comparison of cross-sectional areas in three-phase segmentation

\section{Natural material}

The oil sand specimen used in this study as a natural porous material was scanned using an X-ray CT scanner. The original X-ray CT image of the oil sand is shown in Figure 12(a). Figure 12(b) shows an enlarged view of the part of Figure 12(a) that was used for image segmentation. In the figure, the black regions represent air, and the light-grey regions represent sand grains. The histogram of this image is shown in Figure 13. The histogram has a trimodal distribution, thus implying that this image is of a three-phase material. The lines in the graph indicate the probability distributions of five classes (three for pure voxels and two for mixels) and their superposition. Figure 14 shows the image segmented using the thresholding method based on the mixel model for oil sand. Using the segmented image, the authors calculated the occupied area and occupancy ratio of each constituent in the oil sand (Table 3 ).

The original 3D X-ray CT image of oil sand reconstructed using 40 slices is shown in Figure 15(a). Figure 15(b) shows a 3D image reconstructed by stacking 40 slices segmented using the aforementioned thresholding method, as shown in Figure 14. The legend in Figure 15(b) is the same as that in Figure 14.

\section{Validation of results}

The authors can verify the segmentation method introduced in this study by comparing the cross-sectional area of the thin wires as calculated from the voxel number of the segmented image with their actual cross-sectional area. The ratio of these two values represents the relative error of this method.

For two-phase materials, the relative error in the cross-sectional area of the bundled aluminium wires was $0 \cdot 09$, while that for bundled nylon threads was 0.03 (Table 1 ). These results are in good agreement with the actual cross-sectional areas of the wires and threads used herein. Therefore, the segmentation method is effective for two-phase materials.

For three-phase materials, relative error can be evaluated in a manner similar to that described above. Relative error in the cross-sectional

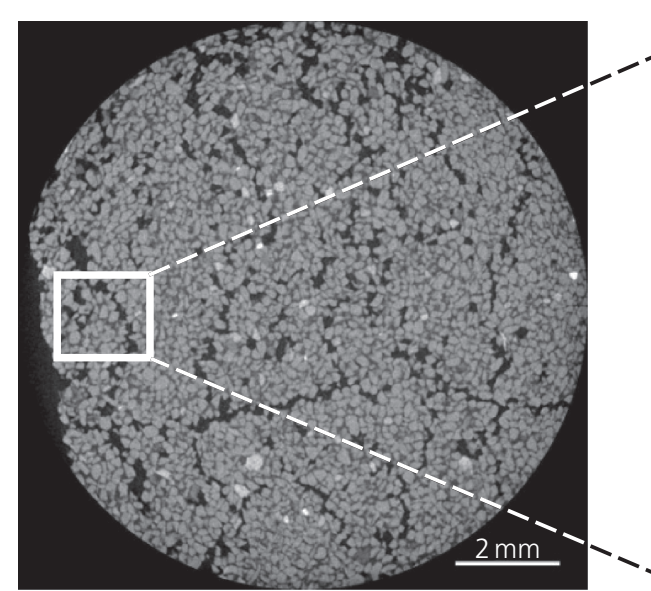

(a)

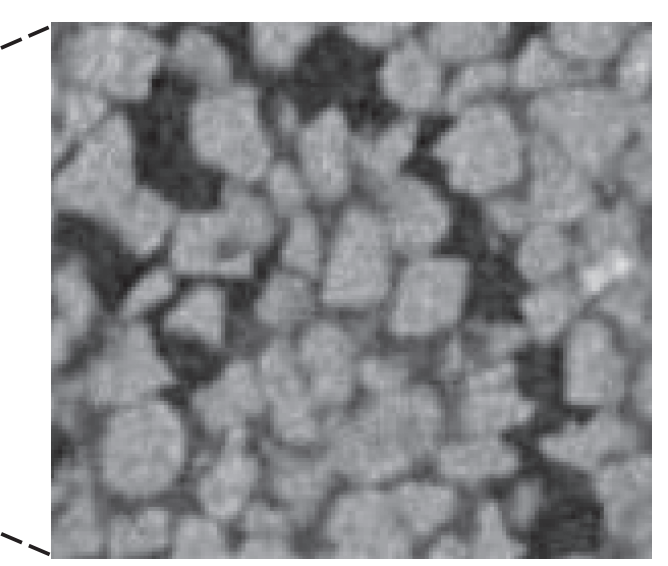

(b)

Figure 12. (a) Original X-ray CT image of oil sand and

(b) enlarged view (size 1.4 × $1.6 \mathrm{~mm}$ ); black regions represent air and light-grey regions represent sand grains 


\section{Offprint provided courtesy of www.icevirtuallibrary.com Author copy for personal use, not for distribution}

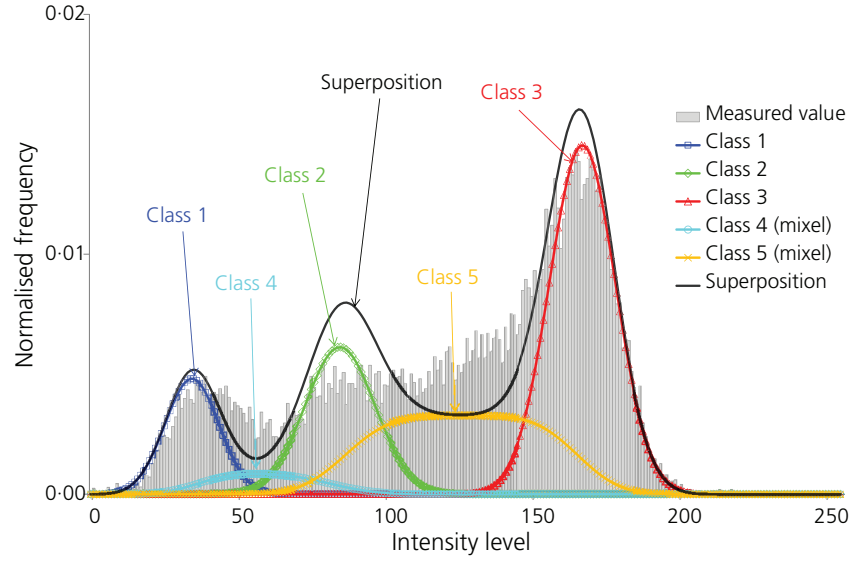

Figure 13. Histogram of CT image of oil sand in Figure 12(b). The lines indicate the probability distributions of five classes and their superposition

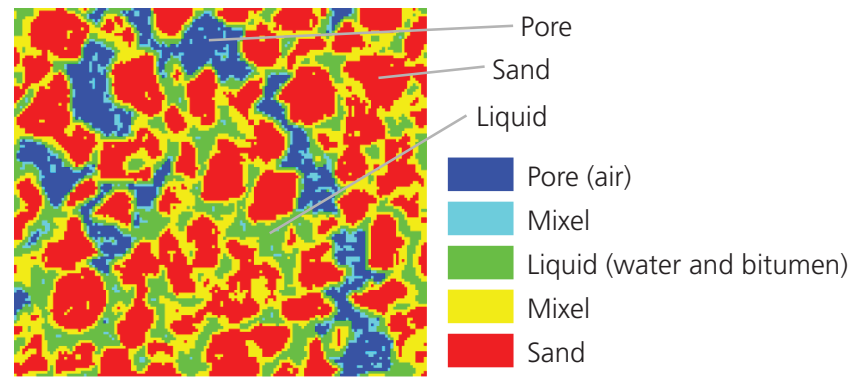

Figure 14. Image segmented using the thresholding method based on the mixel model; blue regions represent class 1 (air in pore), green regions represent class 2 (liquid phase: water and bitumen), red regions represent class 3 (sand grains), sky blue regions represent class 4 (mixel of classes 1 and 2) and yellow regions represent class 5 (mixel of classes 2 and 3 )

\begin{tabular}{lcc}
\hline Constituent & $\begin{array}{c}\text { Occupied } \\
\text { area: } \mathrm{mm}^{2}\end{array}$ & $\begin{array}{c}\text { Occupancy } \\
\text { ratio: \% }\end{array}$ \\
\hline Sand grain & 67.5 & 58.8 \\
Water and bitumen & 32.9 & 28.7 \\
Air & 14.4 & 12.5
\end{tabular}

Table 3. Occupancy ratio of each phase constituting oil sand

area of the ten aluminium wires in the bundle of aluminium wires and nylon threads was 0.04 and that of the ten nylon threads in the same bundle was $0 \cdot 21$ (Table 2). The calculated cross-sectional area of aluminium wires agrees well with the actual cross-sectional area. However, that is not the case with the nylon threads. This is because in the CT image shown in Figure 11, halation occurred

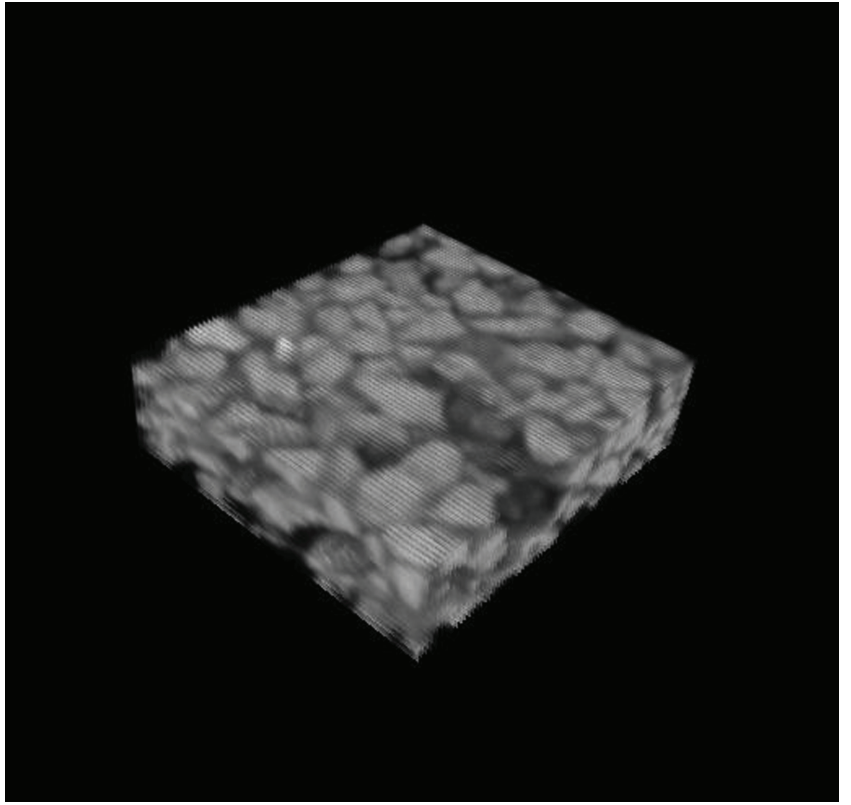

(a)

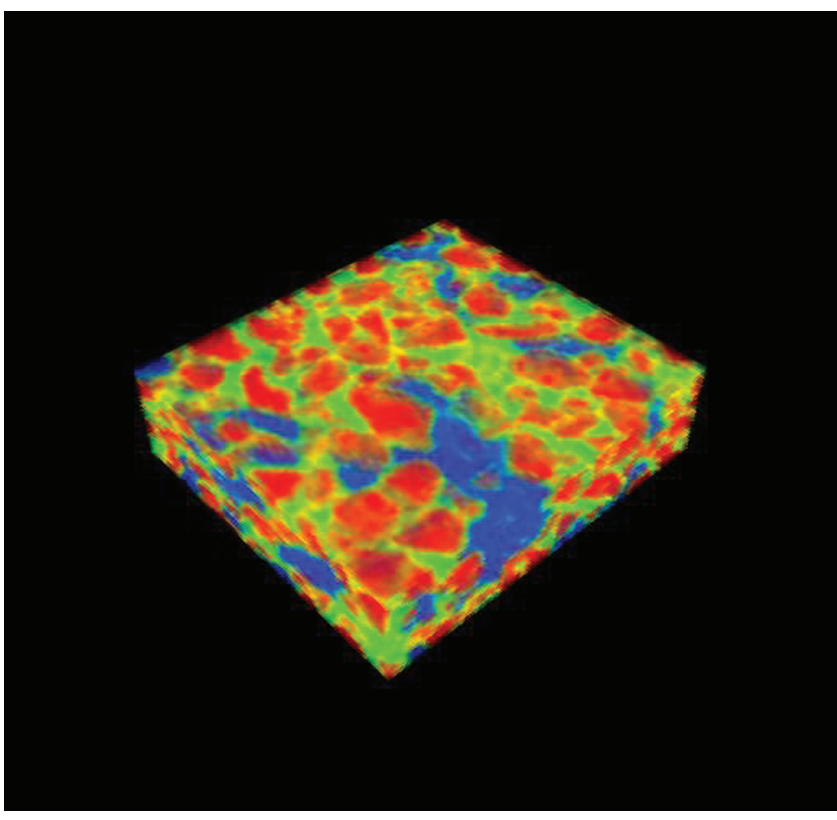

(b)

Figure 15. (a) Original 3-D X-ray CT image of oil sand and (b) 3-D image segmented using the thresholding method based on the mixel model; legend is the same as in Figure 14

against the aluminium wires and strengthened the intensity of the air region, thus resulting in an overestimation of the nylon thread area. However, it is believed that this error can be minimised as the device and the technique are refined.

According to Figure 14, oil sand comprises sand grains, liquid (water and bitumen) and air. The liquid wets the surfaces of the 
sand grains, and air exists in the remainder of the pore volume. The liquid phase could not be sub-classified because the density difference between bitumen and water is small. This is evident from the histogram shown in Figure 13.

Herein, the occupancy ratio, which is the ratio of object phase volume to bulk volume of oil sand, is evaluated. The occupancy ratios of sand grain, liquid (bitumen and water) and air are 58.8\%, $28.7 \%$ and $12 \cdot 5 \%$, respectively (Table 3 ). If bitumen can be separated from liquid, fundamental data for quantifying resources in oil sand formations can be obtained.

Figure 15(b) illustrates not only the arrangement of sand grains but also the pore connectivity network. In the case that a numerical simulation of immiscible fluid behaviour is needed, the geometry and connectivity of pores can be used for effective estimation.

\section{Conclusions}

This paper introduces a thresholding method that considers the partial volume effect for two- and three-phase digital images. The method was verified using X-ray CT images of thin line shapes such as aluminium wires and nylon threads, and then successfully applied to the CT images of oil sand. The segmentation method introduced in the study allows us to estimate the volume and occupancy ratio of each constituent forming a porous material with reasonable accuracy.

A solution to the problem of separating bitumen and water from each other would afford us with fundamental data for quantifying resources in oil sand formation. The geometry and connectivity of pores in oil sand can be used in numerical simulations for estimating the behaviour of immiscible fluids. These are considerations for future work.

\section{Acknowledgements}

Japan Petroleum Exploration Co., Ltd. provided the oil sand sample. Mr H. Yamanaka, a graduate student from Hokkaido University, carried out the required X-ray CT scanning. Furthermore, the authors received constructive comments from anonymous reviewers. Their contributions are appreciated.

\section{Appendix 1: Mixture model for multi-phase objects}

Spatial distribution data of the X-ray attenuation coefficients of a multi-phase object are obtained by X-ray CT scanning. Using these data, a greyscale image is drawn as a mixture of pure pixels, which correspond to pixels covered by a single phase, and mixels, which correspond to pixels occupied by two or more phases (Figure 1). In addition, a histogram of the attenuation coefficient or intensity level of the image is generated. In fact, the histogram of pure pixels follows a normal distribution $N\left(x ; \mu_{i}, \sigma_{i}^{2}\right)$ with the expectation $\mu_{i}$ and variance $\sigma_{i}^{2}$ of constituent class $i$. Accordingly, the PDF $f_{i}(x)$ of constituent class $i$ corresponding to the pure pixels of phase $i$ can be expressed as Equation 1.

For materials with more than two phases, the authors need mixels comprising more than two classes. In reference to Kitamoto (2000), the PDF of an $n$-class mixel for $n_{p}$-phase materials $\left(2 \leq n \leq n_{p}\right)$ can be generalised according to the following equation

$$
\begin{aligned}
M_{i_{1} i_{2} \cdots i_{n}}(x)= & \frac{1}{B\left(m_{1}, m_{2}, \ldots, m_{n}\right)} \int_{0}^{1} \int_{0}^{1} \ldots \int_{0}^{1} a_{i_{1}}^{m_{1}-1} a_{i_{2}}^{m_{2}-1} \ldots \\
& a_{i_{n}-1}^{m_{n}-1} N\left(x ; \mu_{a}, \sigma_{a}^{2}\right) \mathrm{d} a_{i_{1}} \mathrm{~d} a_{i_{2}} \ldots \\
& \mathrm{d} a_{i_{n-1}}\left(i_{1}, i_{2}, \ldots, i_{n} \in 1,2, \ldots, n_{p}\right. \\
& \left.1 \leq i_{1}<i_{2}<\ldots<i_{n} \leq n_{p}\right)
\end{aligned}
$$

12.

where the multi-variate beta function (Letaca et al., 2001; Mauldon, $1959)$ is as follows

13. $B\left(m_{1}, m_{2}, \ldots, m_{n}\right)=\int_{0}^{1} \int_{0}^{1} \ldots \int_{0}^{1} a_{i_{1}}^{m_{1}-1} a_{i_{2}}^{m_{2}-1} \ldots a_{i_{n}}^{m_{n}-1} \mathrm{~d} a_{i_{1}} \mathrm{~d} a_{i_{2}} \ldots \mathrm{d} a_{i_{n-1}}$

where $a_{i}$ is the area proportion of constituent class $i$ and $\sum_{j=i_{1}}^{i_{n}} a_{j}=1$ $\left(a_{j} \geq 0\right)$ holds, and $\mu_{a}$ and $\sigma_{a}^{2}$ are as follows

14. $\mu_{a}=\sum_{j=i_{1}}^{i_{n}} a_{j} \mu_{j}$

15.

$$
\sigma_{a}^{2}=\sum_{j=i_{1}}^{i_{n}} a_{j}^{2} \sigma_{j}^{2}
$$

The total number of class classifications (pure pixels and mixels) in an image of a multi-phase material $N_{n_{p}}$ obeys the relation expressed by the following progression

16. $N_{n_{p}+1}=2\left(N_{n_{p}}+1\right)-1 \quad\left(n_{p}=1,2, \ldots\right)$

Accordingly, the total number of class classifications can be expressed as follows

17. $N_{n_{p}}=2^{n_{p}}-1 \quad\left(n_{p}=1,2, \ldots\right)$

For example, when an image is of a single-phase material, that is, $n_{p}=1, N_{1}$ is apparently 1, calculated using Equation 17 as $N_{1}=2-$ $1=1$. When an image is of a two-phase material, showing its bimodal histogram, that is, $n_{p}=2, N_{2}=2^{2}-1=3$. Repeating this calculation, $N_{3}=2^{3}-1=7$; if $n_{p}=3, N_{4}=2^{4}-1=15$; if $n_{p}=4$, 
$N_{5}=2^{5}-1=31$; if $n_{p}=5$ and so on. Similarly, for images of multiphase materials, the number of pixel classifications increases rapidly as the number of phases in a material increases.

Here, the total number of mixel classifications is obtained by subtracting the number of pure pixel classifications $n_{p}$ from $N_{n_{p}}$, then $2^{n_{p}}-n_{p}-1\left(n_{p}=1,2, \ldots\right)$. In the segmentation carried out in this study, the number of thresholds is double the total number of mixel classifications, resulting in the number $2^{n_{p}+1}-2 n_{p}-2$. Furthermore, the number of thresholds increases rapidly as the number of phases in a material increases.

\section{Appendix 2: Maximum likelihood thresholding criteria}

Let $\tilde{\omega}_{k}$ and $\tilde{\sigma}_{k}^{2}$ be the occurrence probability and variance, respectively, of class $k$. Using the occurrence probability, $\tilde{\omega}_{k}$, and variance, $\tilde{\sigma}_{k}^{2}$, of class $k$, the expectation of variance within each class, $\tilde{\sigma}^{2}$, is expressed as follows

$$
\text { 18. } \tilde{\sigma}^{2}=\sum_{k=1}^{M} \tilde{\omega}_{k} \tilde{\sigma}_{k}^{2}
$$

Here, four types of maximum logarithmic likelihoods are summarised in relation to statistical properties of each class with reference to Sekita et al. (1995). For the first case that $\omega_{k}$ and $\sigma_{k}^{2}$ of each class are almost the same, the following maximum likelihood thresholding criterion was introduced by Otsu (1979)

19. $J_{O}(\mathbf{t})=-\ln \tilde{\sigma}$

For the second case that $\omega_{k}$ is almost the same but $\sigma_{k}^{2}$ is different, Sekita et al. (1995) proposed the following criterion

20.

$$
J_{D}(\mathbf{t})=-\sum_{k=1}^{M} \tilde{\omega}_{k} \ln \tilde{\sigma}_{k}
$$

For the third case that $\omega_{k}$ is different but $\sigma_{k}^{2}$ is almost the same, Kurita et al. (1992) proposed the following criterion

21. $J_{Q}(\mathbf{t})=\sum_{k=1}^{M} \tilde{\omega}_{k} \ln \frac{\tilde{\omega}_{k}}{\tilde{\sigma}}$

For the fourth case that both $\omega_{k}$ and $\sigma_{k}^{2}$ are different, Kitller and Illingworth (1986) proposed the following criterion

22.

$$
J_{K}(\mathbf{t})=\sum_{k=1}^{M} \tilde{\omega}_{k} \ln \frac{\tilde{\omega}_{k}}{\tilde{\sigma}_{k}}
$$

Accordingly, all the above-mentioned maximum likelihood thresholding criteria are determined using both $\tilde{\omega}_{k}$ and $\tilde{\sigma}_{k}^{2}$.

\section{REFERENCES}

Baveye PC, Laba M, Otten W et al. (2010) Observer-dependent variability of the thresholding step in the quantitative analysis of soil images and X-ray microtomography data. Geoderma 157(1-2): 51-63, http://dx.doi.org/10.1016/j. geoderma.2010.03.015.

Bhattad P, Willson CS and Thompson KE (2010) Segmentation of low-contrast three-phase $\mathrm{x}$-ray computed tomography images of porous media. In Advances in Computed Tomography for Geomaterials (GeoX 2010, New Orleans, USA) (Alshibli KA and Reed AH (eds)). London, ISTE, pp. 254-261.

Choi HS, Haynor DR and Kim YM (1991) Partial volume tissue classification of multichannel magnetic-resonance images:amixel model. IEEE Transactions on Medical Imaging 10(3): 395-407, http://dx.doi.org/10.1109/42.97590.

Curry III TS, Dowdey JE and Murry Jr RC (1990) Christensen's Physics of Diagnostic Radiology, 4th edn. Philadelphia, Lea \& Febiger, p. 522.

Houston AN, Otten W, Baveye PC and Hapca S (2013) Adaptivewindow indicator kriging: a thresholding method for computed tomography. Computers and Geosciences 54: 239-248, http://dx.doi.org/10.1016/j.cageo.2012.11.016.

lassonov P, Gebrenegus T and Tuller M (2009) Segmentation of $\mathrm{X}$-ray computed tomography images of porous materials: a crucial step for characterization and quantitative analysis of pore structures. Water Resources Research 45: W09415, http://dx.doi.org/10.1029/2009WR008087.

Kageyama Y and Nishida M (2004) Lineament detection from land cover information in mixels using Landsat-TM data. Electrical Engineering of Japan 148(4): 65-73, http://dx.doi.org/10.1002/ eej.10342.

Kak AC and Slaney M (1988) Principles of Computerized Tomographic Imaging. SIAM, New York, p. 327.

Kato M, Kobayashi Y, Kawasaki S and Kaneko K (2009) Extraction of porosity change of geomaterials using X-ray CT images. Geoinformatics 20(2): 112-113 (in Japanese with English title)

Kato M, Takahashi M and Kaneko K (2008) Estimation of rock porosity using X-ray CT image. Geoinformatics 19(2): 132133 (in Japanese with English title).

Kato M, Takahashi M, Kawasaki K, Mukunoki T and Kaneko K (2013) Evaluation of porosity and its variation in porous materials using microfocus $\mathrm{X}$-ray computed tomography considering the partial volume effect. Materials Transactions 54(9): 1678-1685, http://dx.doi.org/10.2320/ matertrans.M-M2013813.

Kawaragi C, Yoneda T, Sato T and Kaneko K (2009) Microstructure of saturated bentonites characterized by X-ray CT observations. Engineering Geology 106(1-2): 51-57, http://dx.doi.org/10.1016/j.enggeo.2009.02.013.

Ketcham RA and Carlson WD (2001) Acquisition, optimization and interpretation of X-ray computed tomographic 


\section{Offprint provided courtesy of www.icevirtuallibrary.com Author copy for personal use, not for distribution}

imagery: applications to the geosciences. Computers and Geosciences 27(4): 381-400, http://dx.doi.org/10.1016/ S0098-3004(00)00116-3.

Kim JS, Singh V, Lee JK et al. (2005) Automated 3-D extraction and evaluation of the inner and outer cortical surfaces using a Laplacian map and partial volume effect classification. NeuroImage 27(1): 210-221, http://dx.doi.org/10.1016/j. neuroimage.2005.03.036.

Kitamoto A (1999) A maximum likelihood thresholding method considering the effect of mixels. Technical Report of IEICE PRMU99-166, 7-14 (in Japanese with English abstract).

Kitamoto A (2000) The moments of the mixel distribution and its application to statistical image classification. In Advances in Pattern Recognition (Proceedings of the Joint IAPR International Workshops SSPR 2000 and SPR 2000, Alicante, Spain), Lecture Notes in Computer Science 1876. Springer, Berlin/Heidelberg, pp. 521-531, http://dx.doi. org/10.1007/3-540-44522-6.

Kitamoto A and Takagi M (1998a) Estimating the area properties of mixels using mixture density estimation with mixel densities. Denshi Joho Tsushin Gakkai Ronbunshi (Transactions of IEICE) J81-D-II(6): 1160-1172 (in Japanese with title and diagram captions in English).

Kitamoto A and Takagi M (1998b) Image classification method using area proportion density that reflects the internal structure of mixels. Denshi Joho Tsushin Gakkai Ronbunshi (Transactions of IEICE) J81-D-II(11): 2582-2597 (in Japanese with title and diagram captions in English).

Kitamoto A and Takagi M (1999) Image classification using probabilistic models that reflect the internal structure of mixels. Pattern Analysis and Applications 2(1): 31-43, http://dx.doi.org/10.1007/s100440050012.

Kitamoto A and Takagi M (2000) The area proportion distribution-relationship with the internal structure of mixels and its application to image classification. Systems and Computers in Japan 31(5): 57-76, http://dx.doi.org/10.1002/ (SICI)1520-684X(200005).

Kittler J and Illingworth J (1986) Minimum error thresholding. Pattern Recognition 19(1): 41-47, http://dx.doi. org/10.1016/0031-3203(86)90030-0.

Kobayashi Y, Kawasaki S, Kato M, Mukunoki T and Kaneko K (2009) Applicability of a method for evaluation of porosity to biogrouted geomaterials. Journal of MMIJ 125(10,11): 540-546 (in Japanese with English abstract).

Kobayashi Y, Kawasaki S, Kato M, Mukunoki T and Kaneko K (2010) Evaluation of porosity in geomaterials treated with biogrout considering partial volume effect. In Advances in Computed Tomography for Geomaterials (GeoX 2010, New Orleans, USA) (Alshibli KA and Reed AH (eds)). ISTE, London, pp. 287-294.

Kumar M, Lebedeva E, Melean Y et al. (2010) Micropetrophysical experiments via tomography and simulation. In Advances in Computed Tomography for Geomaterials (GeoX 2010, New Orleans, USA) (Alshibli KA and Reed AH (eds)). ISTE, London, pp. 238-253.
Kurita T, Otsu N and Abdelmalek N (1992) Maximum likelihood thresholding based on population mixture models. Pattern Recognition 25(10): 1231-1240, http://dx.doi. org/10.1016/0031-3203(92)90024-D.

Letaca G, Massamb H and Richards D (2001) An expectation formula for the multivariate Dirichlet distribution. Journal of Multivariate Analysis 77(1): 117-137, http://dx.doi. org/10.1006 jmva.2000.1928.

Mauldon JG (1959) A generalization of the beta-distribution. The Annals of Mathematical Statistics 30(2): 509-520, http://dx.doi.org/10.1214/aoms/1177706265.

Oh W and Lindquist WB (1999) Image thresholding by indicator kriging. IEEE Transactions on Pattern Analysis and Machine Intelligence 21(7): 590-602, http://dx.doi. org/10.1109/34.777370.

Okamoto K and Fukuhara M (1996) Estimation of paddy field area using the area ratio of categories in each mixel of Landsat TM. International Journal of Remote Sensing 17(9): 1735 1749, http://dx.doi.org/10.1080/01431169608948736.

Otsu N (1979) A threshold selection method from gray-level histograms. IEEE Transactions on Systems, Man, and Cybernetics SMC-9(1): 62-66, http://dx.doi.org/10.1109/ TSMC.1979.4310076.

Pal NR and Pal SK (1993) A review on image segmentation techniques. Pattern Recognition 26(9): 1277-1294, http://dx.doi.org/10.1016/0031-3203(93)90135-J.

Sahoo PK, Soltani S, Wong AKC and Chen YC (1988) A survey of thresholding techniques. Computer Vision, Graphics, and Image Processing 41(2): 233-260, http://dx.doi. org/10.1016/0734-189X(88)90022-9.

Schlüter S, Weller U and Vogel H-J (2010) Segmentation of $\mathrm{X}$-ray microtomography images of soil using gradient masks. Computers and Geosciences 36: 1246-1251, http://dx.doi. org/10.1016/j.cageo.2010.02.007.

Sekiguchi K (2006) Resources and reserves of heavy oil and extraheavy oil/natural bitumen. Journal of the Japan Institute of Energy 85(4): 258-264 (in Japanese with title and abstract in English).

Sekita I, Kurita T, Otsu N and Abdelmalek NN (1995)

Thresholding methods considering the quantization error of an image. Denshi Joho Tsushin Gakkai Ronbunshi (Transactions of IEICE) J78-D-II(12): 1806-1812 (in Japanese with title and diagram captions in English).

Sezgin M and Sankur B (2004) Survey over image thresholding techniques and quantitative performance evaluation. Journal of Electronic Imaging 13(1): 146-168, http://dx.doi. org/10.1117/1.1631315.

Shattuck DW, Sandor-Leahy SR, Schaper KA, Rottenberg DA and Leahy RM (2001) Magnetic resonance image tissue classification using a partial volume model. NeuroImage 13(5): 856-876, http://dx.doi.org/10.1006/nimg.2000.0730.

Tohka J, Zijdenbos A and Evans A (2004) Fast and robust parameter estimation for statistical partial volume models in brain MRI. NeuroImage 23(1): 84-97, http://dx.doi. org/10.1016/j.neuroimage.2004.05.007. 
Vinegar HJ and Wellington SL (1987) Tomographic imaging of three-phase flow experiments. Review of Scientific Instruments 58(1): 96-107.

Yamanaka H, Kawasaki S, Kato M, Mukunoki T and Kaneko K (2011) Segmentation of internal structure of two-phase materials using micro-focus X-ray CT. Jiban Kogaku Janaru (Japanese Geotechnical Journal) 6(2): 273-284 (in Japanese with English abstract), http://dx.doi.org/10.3208/jgs.6.273.

\section{WHAT DO YOU THINK?}

To discuss this paper, please submit up to 500 words to the editor at journals@ice.org.uk. Your contribution will be forwarded to the author(s) for a reply and, if considered appropriate by the editorial panel, will be published as a discussion in a future issue of the journal. 